\title{
FATORES ASSOCIADOS À BAIXA APTIDÃO FÍSICA DE ADOLESCENTES
}

FACTORS ASSOCIATED WITH LOW PHYSICAL FITNESS IN ADOLESCENTS

FACTORES ASOCIADOS A LA BAJA APTITUD FÍSICA EN ADOLESCENTES

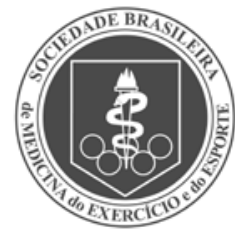

Artigo Original

Original ARTICLE

Artículo Original
Thais Almeida Pereira'

(Educador físico)

Mauren Lúcia de Araújo Bergmann' (Educador físico)

Gabriel Gustavo Bergmann ${ }^{1}$

(Educador físico)

1. Universidade Federal do Pampa (Unipampa), Campus Uruguaiana, RS, Brasil.

\section{Correspondência:}

Gabriel Gustavo Bergmann BR 472, Km 592, Caixa postal 118, Uruguaiana, RS, Brasil. 97500-970. gabrielgbergmann@gmail.com

\section{RESUMO}

Introdução: Os componentes da Aptidão Física Relacionada à Saúde (ApFRS) estão associados a prevenção e redução do desenvolvimento de doenças crônicas, de incapacidades funcionais e de dificuldades na realização de atividades diárias. Objetivo: Identificar a prevalência e os fatores associados à baixa ApFRS em adolescentes. Métodos: Estudo transversal realizado com 1.455 escolares (50,9\% meninas) de 10 a 17 anos, composto por três variáveis dependentes: aptidão cardiorrespiratória (ApC), força/resistência muscular (F/R) e flexibilidade (Flex). Para a medida e classificação da ApFRS (recomendada ou baixa) foi utilizada a bateria de testes com os pontos de corte propostos pelo Projeto Esporte Brasil. As variáveis independentes foram organizadas em três blocos: indicadores sociodemográficos (seis fatores), comportamentais (nove fatores) e antropométricos (três fatores). Para a análise dos dados utilizou-se estatística descritiva, teste de qui-quadrado para heterogeneidade e para tendência e regressão logística binária. O modelo multivariado final considerou apenas fatores com nível de significância inferior a 5\%. Resultados: As prevalências de baixa ApC, F/R e Flex foram de 74,1\% (IC 95\%: 71,6-76,5), 27,4\% (IC 95\%: 25,0-29,7) e 33,1\% (IC 95\%: 30,6-35,5), respectivamente. A análise multivariada indicou que dos 18 fatores analisados, nove foram associados à baixa ApC e cinco, à baixa $F / R$ e à Flex. Apenas a idade (direta com a baixa ApC e F/R e inversa com a baixa Flex) associou-se $(p<0,05)$ aos três componentes da ApFRS. Adolescentes classificados como muito sedentários (ApC e F/R), com tempo de tela de três ou mais horas por dia (ApC e Flex) e com indicadores antropométricos aumentados (os três componentes) apresentam mais chance $(p<0,05)$ de ter baixa ApFRS. Conclusão: Os fatores associados à baixa $A p C$ è baixa F/R muscular são similares, porém, para a baixa Flex os fatores associados tendem a ser outros.

Descritores: aptidão física; exercício; saúde; adolescente; estudos transversais.

\section{ABSTRACT}

Introduction: The Health-Related Physical Fitness (HRPF) components are associated with the prevention and reduction of chronic diseases, functional disabilities, and difficulties in performing daily activities. Objective: To identify the prevalence and associated factors with low HRPF in adolescents. Methods: Cross-sectional study conducted with 1,455 students (50.9\% girls) from 10 to 17 years old, comprising three dependent variables: cardiorespiratory fitness (CRF), muscular strength/endurance (S/E) and flexibility (Flex). For the measurement and classification of HRPF (recommended or low), a battery of tests was used, as well as the cutoff points proposed by Projeto Esporte Brasil. The independent variables were organized into three blocks of indicators: sociodemographic (six factors), behavioral (nine factors), and anthropometric (three factors). Data analysis was done by descriptive statistics, chi-squared test for heterogeneity and trend, and binary logistic regression. The multivariable final model considered only factors with p-value lower than 0.05. Results: The prevalence of low CRF, S/E and Flex was 74.1\% (95\% Cl: 71.6-76.5), 27.4\% (95\% Cl: $25.0-29.7)$ and $33.1 \%$ (95\% Cl: 30.6-35.5), respectively. Multivariate analyses indicated that from the 18 factors analyzed, nine were associated with low CRF, and five with low S/E and Flex. Only the age (directly to low CRF and $S / E$ and inversely to low Flex) was associated $(p<0.05)$ to the three HRPF components. Adolescents classified as very sedentary (CRF and S/E), with screen time of three or more hours per day (CRF and Flex) and with increased anthropometric indicators (three components of HRPF) showed more chance $(p<0.05)$ to present low HRPF. Conclusion: Factors associated with low CRF and S/E are similar. However, for low Flex the associated factors tend to be different.

Keywords: physical fitness; exercise; health; adolescent; cross-sectional studies.

\section{RESUMEN}

Introducción: Los componentes de la aptitud física relacionados con la salud (ApFRS) están asociados con la prevención y reducción del desarrollo de enfermedades crónicas, discapacidades funcionales y dificultades para realizar las actividades diarias. Objetivo: Identificar la prevalencia y los factores asociados con la baja ApFRS en adolescentes. Métodos: Estudio transversal con 1.455 estudiantes (50,9\% niñas) de 10 a 17 años, que consta de tres variables dependientes: aptitud cardiorrespiratoria (ApC), fuerza/resistencia muscular (F/R) y flexibilidad (Flex). Para la medición y clasificación de la ApFRS (recomendada o baja) se utilizó una batería de pruebas con puntos de corte propuestos por el Projeto Esporte Brasil. Las variables independientes fueron organizadas en tres bloques: indicadores sociodemográficos (seis factores), de comportamiento (nueve factores) y antropométricos (tres factores). Para el análisis de los datos se utilizó estadística descriptiva, pruebas de chi-cuadrado para heterogeneidad y tendencia y la regresión logística binaria. 
El modelo multivariable final ha considerado sólo factores con nivel de significación abajo de 5\%. Resultados: La prevalencia de baja ApC, F/R y Flex fue 74, 1\% (IC 95\%: 71,6-76,5), 27,4\% (IC 95\%: 25,0-29,7) y 33,1\% (IC 95\%: 30,6-35,5), respectivamente. El análisis multivariable indicó que de los 18 factores analizados, nueve se asociaron con la baja ApC y cinco con la baja F/R y Flex. Sólo la edad (directamente con la baja ApC y baja F/R y revertida con baja Flex) se asoció $(p<0,05)$ a los tres componentes de ApFRS. Adolescentes clasificados como muy sedentarios (ApC y F/R), con tiempo de pantalla de tres o más horas por día (ApC y Flex) y con aumento de los indicadores antropométricos (tres componentes) tienen más probabilidad ( $p<0,05)$ de tener baja ApFRS. Conclusión: Los factores asociados con la baja ApC y baja F/R son similares; sin embargo, para la baja Flex los factores tienden a ser otros.

Descriptores: aptitud física; ejercicio; salud; adolescente; estudios transversales.

\section{INTRODUÇÃO}

Uma elevada frequência de adolescentes brasileiros não atinge as recomendações de Aptidão Física Relacionada à Saúde (ApFRS) ${ }^{1-8}$. Considerando que inúmeros problemas de saúde estão associados à baixa aptidão física e que seu desenvolvimento muitas vezes inicia na infância ou na adolescência9 , é necessário que este quadro seja revertido.

A identificação de fatores associados à baixa ApFRS pode fornecer informações para que intervenções específicas para distintos grupos sejam elaboradas. Isto proporcionaria alternativas para que estes jovens atingissem melhores níveis de aptidão física e saúde.

Uma série de estudos tem se preocupado em identificar associações entre os componentes da ApFRS e diversos fatores. Dentre eles, indicadores sociodemográficos, como o sexo ${ }^{1-7}$, a faixa etária ${ }^{1-7}$, indicadores socioeconômico ${ }^{1,2,4}$ e a área de domicílio ${ }^{2,7}$; indicadores comportamentais, como comportamento sedentário ${ }^{10-12}$ e inatividade física ${ }^{1,10-12}$; e indicadores antropométricos ${ }^{2,11-14}$. No entanto, poucos são os estudos que associaram em conjunto vários fatores à baixa ApFRS considerando todos os seus componentes. Esta análise é importante, pois os componentes da ApFRS apresentam características específicas e podem se associar diferentemente a cada fator considerado ${ }^{8}$. Este estudo teve por objetivo identificar a prevalência e os fatores sociodemográficos, comportamentais e antropométricos, associados à baixa ApFRS de adolescentes.

\section{MATERIAIS E MÉTODOS}

Este estudo transversal foi realizado com escolares de 10 a 17 anos de escolas públicas das zonas urbana e rural do município de Uruguaiana/RS. O tamanho mínimo da amostra foi calculado de acordo com: a) população de 15.210 escolares desta faixa etária'5; b) prevalência de baixa aptidão física estimada em 50\%; c) intervalo de confiança de 95\% (IC 95\%); d) erro amostral de três pontos percentuais; e e) efeito de delineamento igual a 1,5. Com a adoção destes critérios foi estimada a necessidade de avaliar 1.495 escolares.

O critério de amostragem adotado foi probabilístico por conglomerados, onde cada escola foi considerada um conglomerado. Uma escola da zona rural e nove escolas da zona urbana foram selecionadas. Todos os alunos das escolas sorteadas, dentro da faixa etária de interesse, foram convidados a participar do estudo. Os critérios de inclusão considerados foram: a) estar matriculado na rede pública de ensino do município; b) estar dentro da faixa etária proposta; e, c) apresentar o Termo de Consentimento Livre e Esclarecido assinado por um responsável e manifestar interesse em participar. O critério de exclusão considerado foi possuir alguma limitação física e/ou cognitiva que pudesse comprometer o resultado de alguma medida. O estudo foi aprovado pelo Comitê de Ética em pesquisa da instituição onde o estudo foi conduzido (protocolo 042/2010).
A ApFRS foi considerada a partir da aptidão cardiorrespiratória (ApC) (teste de corrida/caminhada de nove minutos); força/resistência muscular (F/R) (teste de abdominais em um minuto); e flexibilidade (Flex) (teste de sentar e alcançar). Para a medida destes componentes e para a classificação dos resultados (recomendada ou baixa), foram utilizados a bateria de testes e os pontos de corte propostos pelo Projeto Esporte Brasil 15 .

As variáveis independentes foram organizadas em três blocos.

Indicadores sociodemográficos: a) o nível socioeconômico (conforme o Critério de Classificação Econômica Brasil ${ }^{16}$ - dividido em cinco níveis, de "A" a "E" - para as análises estatísticas, as categorias A e B, e $D$ e E, foram agrupadas em A/B e D/E); b) a escolaridade do chefe da família em anos ( $\leq 8$ anos ou $>8$ anos); c) a zona de residência (urbana ou rural); d) o tipo de residência (casa ou apartamento); e) o sexo (masculino ou feminino); e f) a idade (anos completos).

Indicadores comportamentais: a) deslocamento até a escola (ativo ou passivo); b) prática de educação física (EF) escolar (sim ou não); c) práticas corporais orientadas além da EF escolar ( $\leq 1$ vez ou $\geq 2$ vezes por semana); d) gostar das aulas de EF (sim ou não); e) gostar de praticar atividades física (sim ou não); f) tempo de tela [tempo utilizado com televisão, jogos eletrônicos e computador ( $\leq 3$ horas ou $>3$ horas)]; g) uso de tabaco e bebida alcoólica (nunca, já usei mas não uso mais ou uso regularmente); e h) nível de atividade física (NAF), estimado pelo Physical Activity Questionnaire for Older Children $\mathrm{PAQ}-\mathrm{C}^{17}$ and $\mathrm{Ado}$ lescents $P A Q-A^{18}$. O resultado (escore) de cada indivíduo foi classificado em muito sedentário, sedentário, moderadamente ativo, ativo ou muito ativo, de acordo com a proposta original $1^{17,18}$.

Indicadores antropométricos: a) Índice de Massa Corporal (IMC), calculado com base na massa corporal em quilogramas (kg) e estatura em metros $(\mathrm{m})$ de cada aluno através da equação IMC = massa corporal $(\mathrm{kg})$ x estatura $\left(\mathrm{m}^{2}\right)$. Os resultados foram classificados conforme a proposta de Cole et al. ${ }^{19}$, b) Circunferência da Cintura (CC), mensurada na região de menor circunferência abdominal, com uma fita métrica flexível, com resolução de 0,1 centímetro, classificada em recomendada ou acima do recomendado ${ }^{20}$, e c) adiposidade corporal, estimada por meio do somatório das dobras cutâneas tricipital e subescapular, classificada em recomendada ou acima do recomendado, de acordo com a proposta do Physical Best ${ }^{21}$.

Todas as medidas foram realizadas por uma equipe de avaliadores (professores e bolsistas/estudantes de educação física) que passaram por uma capacitação. Para a coleta das informações relativas aos indicadores sociodemográficos e comportamentais foi utilizado um questionário aplicado em sala de aula. Os indicadores antropométricos foram medidos individualmente logo após a aplicação do questionário em uma sala na própria escola. Os componentes da ApFRS foram medidos nos ginásios e quadras esportivas das escolas. A coleta de dados ocorreu entre os meses de maio e novembro de 2011. 


\section{Análise estatística}

Para a descrição das variáveis foram utilizadas frequências absolutas e relativas seguidas pelo cálculo do intervalo de confiança de 95\% (IC95\%). Para a descrição da frequência de baixa ApFRS conforme as variáveis independentes analisadas foram utilizados os testes Qui-quadrado para heterogeneidade e tendência. Para a análise multivariável entre os componentes da ApFRS (recomendada/baixa) e as variáveis independentes foi utilizada a regressão logística binária. A entrada de cada uma das variáveis independentes nesta análise ocorreu de acordo com os blocos anteriormente descritos (sociodemográficos, comportamentais e antropométricos). O modelo multivariável final considerou como fatores associados à baixa ApFRS, as variáveis independentes que apresentaram valor-p inferior a 0,05. Todas as análises foram realizadas no pacote estatístico SPSS versão 20.0.

\section{RESULTADOS}

Do total de escolares selecionados, 1.463 foram avaliados. Oito escolares foram excluídos por estarem fora da faixa etária estudada, totalizando 1.455 adolescentes. A Tabela 1, apresenta a descrição dos componentes da ApFRS e das variáveis independentes analisadas.

A frequência de adolescentes classificados com baixa ApFRS de acordo com as variáveis independentes é apresentada na Tabela 2. Com exceção do uso de tabaco, as demais variáveis independentes analisadas apresentaram associação $(p<0,05)$ com a baixa ApC. Das 18 variáveis independentes analisadas 10 e seis, respectivamente, apresentaram associação estatisticamente significativa $(p<0,05)$ com a baixa F/R e baixa Flex.

Na Tabela 3, são apresentados os resultados da regressão logística. Dentre os três desfechos analisados, a baixa ApC, teve o maior número de fatores associados (nove) na análise multivariável. Dentre os 18 fatores analisados, (cinco) se associaram à baixa F/R e à baixa Flex. Apenas a idade apresentou associação com os três desfechos. Porém, indivíduos com idades mais avançadas apresentaram mais chance de terem baixa ApC e baixa F/R, enquanto aqueles com idades menos avançadas apresentam mais chance de terem baixa Flex. Entre os indicadores comportamentais analisados, adolescentes que relataram um tempo de tela diário superior a três horas (ApC e Flex), e que foram classificados como muito sedentários (ApC e F/R) tiveram chance significativamente maior de não atingir as recomendações para a ApFRS. Dentre os indicadores antropométricos, indivíduos com sobrepeso (ApC), com CC aumentada (F/R e Flex) e com adiposidade corporal aumentada (ApC), apresentaram chance significativamente superior de ter baixa ApFRS.

\section{DISCUSSÃO}

O presente estudo objetivou identificar a prevalência e os fatores sociodemográficos, comportamentais e antropométricos associados à baixa ApFRS, configurando-se como o primeiro estudo, a testar associações brutas e ajustadas com este conjunto de variáveis. Esta característica se constitui como a principal contribuição do estudo para a melhor compreensão da temática. Além disto, o tamanho da amostra e o seu procedimento de seleção são outras vantagens deste estudo, pois, fortalecem sua validade externa.

Dentre os três componentes motores da ApFRS, a ApC foi o que apresentou a maior prevalência de adolescentes que não atenderam às recomendações, semelhante aos estudos de Nogueira e Pereira ${ }^{3}$ e Pelegrini et al. ${ }^{6}$ que utilizaram os mesmos procedimentos de medida e classificação da ApFRS que o presente estudo. No entanto, a maior parte dos estudos encontrou maior prevalência de indivíduos que não atenderam às recomendações para a $F / R^{1,2,4,5,7,8}$. Esta diferença pode estar associada aos distintos testes e critérios de classificação da ApFRS utilizados. Contudo, independente do componente que apresentou a maior prevalência de
Tabela 1. Descrição das variáveis analisadas em adolescentes de 10 a 17 anos. Uruguaiana/RS, 2011.

\begin{tabular}{|c|c|c|}
\hline Variável & $\mathbf{N}$ & $\%(\mathrm{IC} 95 \%)$ \\
\hline \multicolumn{3}{|c|}{$\begin{array}{l}\text { Dependente } \\
\end{array}$} \\
\hline \multicolumn{3}{|c|}{ Aptidão Cardiorrespiratória } \\
\hline Abaixo do recomendado & 926 & $74,1(71,6-76,5)$ \\
\hline Recomendado & 324 & $25,9(23,4-28,3)$ \\
\hline \multicolumn{3}{|l|}{ Força/resistência muscular } \\
\hline Abaixo do recomendado & 369 & $27,4(25,0-29,7)$ \\
\hline Recomendado & 976 & $72,6(70,2-74,9)$ \\
\hline \multicolumn{3}{|l|}{ Flexibilidade } \\
\hline Abaixo do recomendado & 451 & $33,1(30,6-35,5)$ \\
\hline Recomendado & 913 & $66,9(64,4-69,3)$ \\
\hline \multicolumn{3}{|c|}{ Indicadores sociodemográficos } \\
\hline \multicolumn{3}{|c|}{\begin{tabular}{l|l} 
Sexo &
\end{tabular}} \\
\hline Masculino & 714 & $49,1(46,5-51,6)$ \\
\hline Feminino & 741 & $50,9(48,3-53,4)$ \\
\hline \multicolumn{3}{|l|}{ Idade } \\
\hline 10 anos & 79 & $5,4(5,1-5,6)$ \\
\hline 11 anos & 209 & $14,4(12,5-16,2)$ \\
\hline 12 anos & 205 & $14,1(12,3-15,8)$ \\
\hline 13 anos & 232 & $15,9(14,0-17,7)$ \\
\hline 14 anos & 211 & $14,5(12,6-16,3)$ \\
\hline 15 anos & 221 & $15,2(13,3-17,0)$ \\
\hline 16 anos & 174 & $12,0(10,3-13,6)$ \\
\hline 17 anos & 124 & $8,5(8,3-8,6)$ \\
\hline \multicolumn{3}{|l|}{ Nível Socioeconômico } \\
\hline Classe D/E & 198 & $15,5(13,5-17,4)$ \\
\hline Classe C & 817 & $63,9(61,2-66,5)$ \\
\hline Classe B & 263 & $20,6(18,3-22,8)$ \\
\hline Escolaridade do chefe da fam & & \\
\hline$\leq$ oito anos & 650 & $47,4(44,7-50,0)$ \\
\hline >oito anos & 721 & $52,6(49,9-55,2)$ \\
\hline Zona de moradia & & \\
\hline Urbana & 1.296 & $90,8(89,3-92,2)$ \\
\hline Rural & 131 & $9,2(9,0-9,3)$ \\
\hline Tipo de residência & & \\
\hline Casa & 1.345 & $94,1(92,8-95,3)$ \\
\hline Apartamento & 84 & $5,9(5,6-6,1)$ \\
\hline Indicadores co & & \\
\hline Deslocamento para a escol & & \\
\hline Ativo & 1.082 & $75,7(73,4-77,9)$ \\
\hline Passivo & 348 & $24,3(22,0-26,5)$ \\
\hline Prática de EF escolar & & \\
\hline Sim & 1.191 & $84,6(82,7-86,4)$ \\
\hline Não & 216 & $15,4(13,5-17,2)$ \\
\hline Práticas corporais orientadas além da & & \\
\hline$\leq 1 \times$ por semana & 1.059 & $77,6(75,3-79,8)$ \\
\hline$\geq 2 x$ por semana & 305 & $22,4(20,1-24,6)$ \\
\hline Gosta das aulas de EF & & \\
\hline $\operatorname{Sim}$ & 1.009 & $79,2(76,9-81,4)$ \\
\hline Não & 265 & $20,8(18,5-23,0)$ \\
\hline Gosta de praticar atividade fís & & \\
\hline Sim & 1.251 & $87,5(85,7-89,2)$ \\
\hline Não & 178 & $12,5(10,7-14,2)$ \\
\hline Tempo de tela & & \\
\hline$\leq 3$ horas & 521 & $35,8(33,3-38,2)$ \\
\hline$>3$ horas & 934 & $64,2(61,7-66,6)$ \\
\hline Uso de tabaco & & \\
\hline Nunca & 1.283 & $89,6(88,0-91,1)$ \\
\hline Já usei, mas não uso mais & 123 & $8,6(8,4-8,7)$ \\
\hline Uso regularmente & 26 & $1,8(1,6-1,9)$ \\
\hline Uso de bebida alcoólica & & \\
\hline Nunca & 803 & $56,1(53,5-58,6)$ \\
\hline Já usei, mas não uso mais & 336 & $23,5(21,3-25,6)$ \\
\hline Uso regularmente & 293 & $20,5(18,4-22,5)$ \\
\hline Nível de atividade física & & \\
\hline Muito sedentário & 360 & $26,8(24,4-29,1)$ \\
\hline Sedentário & 553 & $41,2(38,5-43,8)$ \\
\hline Moderadamente ativo & 318 & $23,7(21,4-25,9)$ \\
\hline Ativo & 112 & $8,3(8,0-8,5)$ \\
\hline Indicadores a & & \\
\hline Índice de massa corporal & & \\
\hline Peso normal & 990 & $72,2(69,8-74,5)$ \\
\hline Sobrepeso & 273 & $19,9(17,7-22,0)$ \\
\hline Obesidade & 109 & $7,9(7,6-8,1)$ \\
\hline Circunferência da cintura & & \\
\hline Recomendada & 1.154 & $79,3(77,2-81,3)$ \\
\hline Aumentada & 301 & $20,7(18,6-22,7)$ \\
\hline Adiposidade corporal & & \\
\hline Recomendada & 999 & $73,5(71,1-75,8)$ \\
\hline Aumentada & 360 & $26,5(24,1-28,8)$ \\
\hline
\end{tabular}




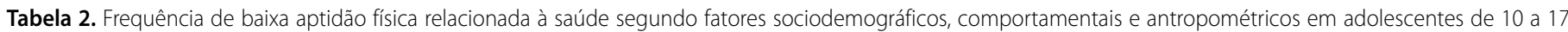
anos. Uruguaiana/RS, 2011

\begin{tabular}{|c|c|c|c|c|c|c|}
\hline & Baixa ApC & $p$ & Baixa F/R & $p$ & Baixa Flex & $\mathbf{P}$ \\
\hline & $\mathrm{N}(\%)$ & & $\mathrm{N}(\%)$ & & $\mathrm{N}(\%)$ & \\
\hline \multicolumn{7}{|c|}{ Indicadores sociodemográficos } \\
\hline Sexo & & 0,000 & & 0,457 & & 0,000 \\
\hline Masculino & $453(68,3)$ & & $191(28,3)$ & & $261(38,4)$ & \\
\hline Feminino & $473(80,6)$ & & $178(26,5)$ & & $190(27,8)$ & \\
\hline Idade (anos) & & 0,000 & & 0,000 & & 0,000 \\
\hline 10 & $14(34,1)$ & & $14(18,2)$ & & $28(36,4)$ & \\
\hline 11 & $132(65,0)$ & & $31(15,0)$ & & $90(43,1)$ & \\
\hline 12 & $130(69,5)$ & & $41(20,4)$ & & $79(39,3)$ & \\
\hline 13 & $158(72,5)$ & & $57(24,9)$ & & $72(31,7)$ & \\
\hline 14 & $143(74,5)$ & & $60(30,6)$ & & $53(26,8)$ & \\
\hline 15 & $131(79,4)$ & & $56(30,4)$ & & $57(30,3)$ & \\
\hline 16 & $129(87,8)$ & & $56(37,1)$ & & $36(23,4)$ & \\
\hline 17 & $89(91,8)$ & & $54(54,0)$ & & $36(32,7)$ & \\
\hline Nível Socioeconômico & & 0,002 & & 0,140 & & 0,605 \\
\hline Classe D/E & $119(71,3)$ & & $54(29,8)$ & & $59(31,9)$ & \\
\hline Classe C & $505(72,0)$ & & $206(27,3)$ & & $248(32,5)$ & \\
\hline Classe B & $194(84,0)$ & & $58(23,6)$ & & $85(34,1)$ & \\
\hline Escolaridade do chefe da família & & 0,000 & & 0,015 & & 0,754 \\
\hline soito anos & $377(67,3)$ & & $144(23,7)$ & & $198(32,5)$ & \\
\hline >oito anos & $494(80,1)$ & & $196(29,8)$ & & $224(33,3)$ & \\
\hline Zona de moradia & & 0,012 & & 0,010 & & 0,001 \\
\hline Urbana & $848(75,2)$ & & $314(26,3)$ & & $383(31,7)$ & \\
\hline Rural & $63(63,6)$ & & $47(37,0)$ & & $59(46,5)$ & \\
\hline Tipo de moradia & & 0,012 & & 0,136 & & 0,646 \\
\hline Casa & $852(73,4)$ & & $334(26,8)$ & & $415(32,9)$ & \\
\hline Apartamento & $60(87,0)$ & & $26(34,7)$ & & $28(35,4)$ & \\
\hline \multicolumn{7}{|c|}{ Indicadores comportamentais } \\
\hline Deslocamento até a escola & & 0,047 & & 0,151 & & 0,399 \\
\hline Ativo & $688(72,8)$ & & $264(26,3)$ & & $329(32,4)$ & \\
\hline Passivo & $225(78,7)$ & & $97(30,4)$ & & $114(35,0)$ & \\
\hline Prática de EF escolar & & 0,000 & & 0,000 & & 0,547 \\
\hline Sim & $743(71,6)$ & & $279(25,0)$ & & $371(33,0)$ & \\
\hline Não & $155(88,1)$ & & $72(37,9)$ & & $69(35,2)$ & \\
\hline Práticas corporais orientadas além da EF escolar & & 0,007 & & 0,060 & & 0,233 \\
\hline$\leq 1 \times$ por semana & $682(75,6)$ & & $272(27,8)$ & & $331(33,4)$ & \\
\hline$\geq 2 x$ por semana & $181(67,3)$ & & $63(22,2)$ & & $85(29,6)$ & \\
\hline Gosta das aulas de EF & & 0,000 & & 0,001 & & 0,918 \\
\hline $\operatorname{Sim}$ & $615(69,4)$ & & $230(24,3)$ & & $317(33,1)$ & \\
\hline Não & $182(86,7)$ & & $81(34,6)$ & & $80(33,5)$ & \\
\hline Gosta de praticar atividade física & & 0,002 & & 0,020 & & 0,087 \\
\hline $\operatorname{Sim}$ & $794(72,9)$ & & $306(26,2)$ & & $382(32,2)$ & \\
\hline Não & $122(84,7)$ & & $54(35,1)$ & & $61(39,1)$ & \\
\hline Tempo de tela & & 0,000 & & 0,655 & & 0,045 \\
\hline$\leq 3$ horas & $300(67,6)$ & & $129(26,7)$ & & $145(29,7)$ & \\
\hline$>3$ horas & $626(77,7)$ & & $240(27,8)$ & & $306(35,0)$ & \\
\hline Uso de tabaco & & 0,040 & & 0,360 & & 0,739 \\
\hline Nunca & $810(73,3)$ & & $810(73,3)$ & & $403(33,4)$ & \\
\hline Já usei, mas não uso mais & $85(78,7)$ & & $85(78,7)$ & & $35(31,0)$ & \\
\hline Uso regularmente & $20(95,2)$ & & $20(95,2)$ & & $6(27,3)$ & \\
\hline Uso de bebida alcoólica & & 0,001 & & 0,086 & & 0,986 \\
\hline Nunca & $499(70,5)$ & & $191(25,1)$ & & $255(33,1)$ & \\
\hline Já usei, mas não uso mais & $219(76,3)$ & & $89(28,4)$ & & $102(32,7)$ & \\
\hline Uso regularmente & $197(82,4)$ & & $80(32,0)$ & & $87(33,3)$ & \\
\hline Nível de atividade física & & 0,000 & & 0,000 & & 0,408 \\
\hline Muito sedentário & $255(86,7)$ & & $121(38,1)$ & & $104(32,2)$ & \\
\hline Sedentário & $353(73,5)$ & & $119(23,1)$ & & $159(30,3)$ & \\
\hline Moderadamente ativo & $187(66,3)$ & & $73(24,3)$ & & $106(35,1)$ & \\
\hline Ativo & $62(57,9)$ & & $21(19,4)$ & & $37(33,9)$ & \\
\hline \multicolumn{7}{|c|}{ Indicadores antropométricos } \\
\hline Índice de massa corporal & & 0,000 & & 0,000 & & 0,039 \\
\hline Peso normal & $619(68,2)$ & & $220(22,8)$ & & $312(31,8)$ & \\
\hline Sobrepeso & $206(86,9)$ & & $95(35,2)$ & & $94(34,6)$ & \\
\hline Obesidade & $96(98,0)$ & & $51(49,0)$ & & $45(41,7)$ & \\
\hline Circunferência da cintura & & 0,000 & & 0,000 & & 0,001 \\
\hline Recomendada & $747(70,7)$ & & $276(24,4)$ & & $357(31,2)$ & \\
\hline Aumentada & $179(92,3)$ & & $93(43,3)$ & & $94(42,7)$ & \\
\hline Adiposidade corporal & & 0,000 & & 0,000 & & 0,052 \\
\hline Recomendada & $613(67,3)$ & & $226(23,1)$ & & $312(31,5)$ & \\
\hline Aumentada & $299(92,6)$ & & $139(39,7)$ & & $133(37,2)$ & \\
\hline
\end{tabular}


Tabela 3. Associação ajustada entre fatores sociodemográficos, comportamentais e antropométricos e a baixa aptidão física relacionada à saúde em adolescentes de 10 a 17 anos. Uruguaiana/RS, 2011.

\begin{tabular}{|c|c|c|c|}
\hline & Baixa ApC & Baixa F/R & Baixa Flex \\
\hline & \begin{tabular}{|c|}
$\begin{array}{c}\text { OR ajustada } \\
\text { (IC } 95 \%)\end{array}$ \\
\end{tabular} & $\begin{array}{c}\text { OR ajustada } \\
\text { (IC95\%) }\end{array}$ & $\begin{array}{l}\text { OR ajustada } \\
\text { (IC95\%) }\end{array}$ \\
\hline \multicolumn{4}{|c|}{ Indicadores sociodemográficos } \\
\hline \multicolumn{4}{|c|}{ 更 } \\
\hline Masculino & 1 & 1 & 1 \\
\hline Feminino & $1,94(1,45-2,59)$ & $0,92(0,71-1,21)$ & $0,60(0,47-0,77)$ \\
\hline \multicolumn{4}{|l|}{ Idade } \\
\hline & $1,30(1,20-1,40)$ & $1,29(1,21-1,39)$ & $0,88(0,83-0,94)$ \\
\hline \multicolumn{4}{|l|}{ Nível socioeconômico } \\
\hline Classe D/E & 1 & 1 & 1 \\
\hline Classe C & $1,03(0,68-1,56)$ & $0,94(0,63-1,40)$ & $1,07(0,73-1,55)$ \\
\hline Classe B & $1,83(1,03-3,25)$ & $0,79(0,47-1,31)$ & $1,09(0,68-1,76)$ \\
\hline \multicolumn{4}{|l|}{$\begin{array}{l}\text { Escolaridade do chefe } \\
\text { da família }\end{array}$} \\
\hline$\leq$ oito anos & 1 & 1 & 1 \\
\hline >oito anos & $1,66(1,20-2,29)$ & $1,40(1,04-1,89)$ & $1,05(0,79-1,39)$ \\
\hline \multicolumn{4}{|l|}{ Zona de moradia } \\
\hline Urbana & $1,26(0,77-2,07)$ & $0,55(0,35-0,86)$ & $0,53(0,35-0,80)$ \\
\hline Rural & 1 & 1 & 1 \\
\hline \multicolumn{4}{|l|}{ Tipo de moradia } \\
\hline Casa & 1 & 1 & 1 \\
\hline Apartamento & $1,50(0,71-3,16)$ & $1,34(0,78-2,28)$ & $1,20(0,72-2,00)$ \\
\hline \multicolumn{4}{|c|}{ Indicadores comportamentais } \\
\hline \multicolumn{4}{|c|}{\begin{tabular}{|l|l|l} 
Deslocamento até a escola & & \\
\end{tabular}} \\
\hline Ativo & 1 & 1 & 1 \\
\hline Passivo & $0,99(0,63-1,57)$ & $1,09(0,72-1,64)$ & $0,70(0,48-1,04)$ \\
\hline \multicolumn{4}{|l|}{ Prática de EF escolar } \\
\hline Sim & 1 & 1 & 1 \\
\hline Não & $0,56(0,23-1,36)$ & $0,74(0,40-1,39)$ & $1,12(0,61-2,06)$ \\
\hline \multicolumn{4}{|c|}{\begin{tabular}{c|c} 
Prática corporal com & \\
orientação além da EF escolar & \\
\end{tabular}} \\
\hline$\leq 1 \times$ por semana & $1,80(0,60-5,34)$ & $1,40(0,54-3,62)$ & $1,10(0,43-2,81)$ \\
\hline$\geq 2 x$ por semana & 1 & 1 & 1 \\
\hline \multicolumn{4}{|l|}{ Gosta das aulas de EF } \\
\hline Sim & 1 & 1 & 1 \\
\hline Não & $2,35(1,12-4,94)$ & $1,40(0,82-2,38)$ & $1,21(0,72-2,04)$ \\
\hline \multicolumn{4}{|l|}{$\begin{array}{l}\text { Gosta de praticar } \\
\text { atividade física }\end{array}$} \\
\hline $\operatorname{Sim}$ & 1 & 1 & 1 \\
\hline Não & $1,64(0,89-3,04)$ & $1,03(0,62-1,71)$ & $1,15(0,71-1,83)$ \\
\hline \multicolumn{4}{|l|}{ Tempo de tela } \\
\hline$\leq 3$ horas & 1 & 1 & 1 \\
\hline$\geq 3$ horas & $1,73(1,24-2,42)$ & $1,04(0,74-1,45)$ & $1,54(1,13-2,10)$ \\
\hline \multicolumn{4}{|l|}{ Uso de tabaco } \\
\hline Nunca & 1 & \begin{tabular}{|c|}
1 \\
\end{tabular} & \begin{tabular}{|c|}
1 \\
\end{tabular} \\
\hline Já usei, mas não uso mais & $0,61(0,32-1,17)$ & $1,06(0,58-1,92)$ & $0,85(0,47-1,55)$ \\
\hline Uso reqularmente & $2,97(0,35-24,68)$ & $2,05(0,68-6,14)$ & $0,55(0,14-2,13)$ \\
\hline Uso de bebida alcoólica & & & \\
\hline Nunca & 1 & 1 & 1 \\
\hline Já usei, mas não uso mais & $0,95(0,63-1,44)$ & $1,04(0,70-1,52)$ & $1,06(0,74-1,52)$ \\
\hline Uso regularmente & $0,64(0,37-1,09)$ & $0,59(0,36-0,97)$ & $1,56(0,99-2,46)$ \\
\hline Nível de atividade física & & & \\
\hline Muito sedentário & $2,05(1,03-4,08)$ & $2,32(1,09-4,91)$ & $1,08(0,58-2,00)$ \\
\hline Sedentário & $1,55(0,90-2,66)$ & $1,40(0,71-2,75)$ & $0,86(0,50-1,46)$ \\
\hline Moderadamente ativo & $1,52(0,87-2,65)$ & $1,42(0,71-2,83)$ & $1,06(0,61-1,81)$ \\
\hline Ativo & 1 & 1 & 1 \\
\hline & dores antropom & étricos & \\
\hline Índice de massa corporal & & & \\
\hline Peso normal & 1 & 1 & 1 \\
\hline Sobrepeso & $1,96(1,00-3,84)$ & $1,33(0,76-2,32)$ & $0,59(0,35-1,02)$ \\
\hline Obesidade & $4,31(0,79-23,40)$ & $1,82(0,78-4,24)$ & $0,45(0,19-1,02)$ \\
\hline Circunferência da cintura & & & \\
\hline Recomendada & 1 & 1 & \begin{tabular}{|c|}
1 \\
\end{tabular} \\
\hline Aumentada & $1,72(0,67-4,40)$ & $1,96(1,04-3,69)$ & $2,33(1,27-4,25)$ \\
\hline Adiposidade corporal & & & \\
\hline Recomendada & 1 & \begin{tabular}{|c|}
1 \\
\end{tabular} & 1 \\
\hline Aumentada & $3,50(1,75-6,99)$ & $1,59(0,97-2,61)$ & $1,40(0,87-2,26)$ \\
\hline
\end{tabular}

indivíduos que não atenderam às recomendações, as prevalências são elevadas nos três componentes motores da ApFRS em todos os estudos. Para a melhor compreensão destas elevadas prevalências e para que estratégias com o intuito de reverter este quadro sejam elaboradas, diferentes fatores associados à baixa ApFRS devem ser considerados.

Entre os indicadores sociodemográficos analisados, o sexo se associou com a baixa ApC e com baixa Flex, e a idade com os três componentes da ApFRS. Porém, adolescentes do sexo feminino e com idades mais avançadas apresentaram mais chance de não atenderem às recomendações para $A p C$ e F/R, enquanto indivíduos do sexo masculino e com menor idade para Flex. Em um estudo realizado com adolescentes desta população ${ }^{22}$, foi encontrado que as meninas e os indivíduos com idades mais avançadas apresentam NAF mais baixos que os meninos e do que os indivíduos de menor idade. Isto pode ajudar a explicar os resultados encontrados para as associações entre sexo, idade e a baixa ApC e a baixa F/R.

Para a os resultados encontrados referentes à baixa Flex, por outro lado, características biológicas talvez exerçam mais influência que comportamentais. As meninas, independentemente da idade, parecem ser mais flexíveis que os meninos ${ }^{3,6}$, podendo explicar o resultado associado ao sexo. Quanto à idade, provavelmente por questões relativas ao rápido crescimento corporal, a Flex tende a diminuir no início da adolescência voltando a aumentar e estabilizar em idades mais avançadas deste período ${ }^{23}$.

Outras três variáveis sociodemográficas apresentaram associação com os componentes da ApFRS. O nível socioeconômico com a ApC, a escolaridade do chefe da família com a ApC e F/R, e a zona de moradia com a F/R e com a Flex. Similar ao encontrado por Guedes et al. ${ }^{1}$ e Silva et al. ${ }^{12}$, no presente estudo, adolescentes provenientes de famílias com maior poder aquisitivo e que os pais possuam maior escolaridade apresentaram mais chance de não atenderem as recomendações para a ApC e F/R. Resultados contrários foram encontrados nos estudos de Jiménez Pavón et al. ${ }^{24}$. Já no estudo de Petroski et al. ${ }^{2}$ não foram encontradas associações significativas.

Embora indicadores socioeconômicos sejam considerados como possíveis fatores associados aos níveis de ApFRS de crianças e adolescentes, estas associações não tem sido consistentes 1,2,24,25. Os resultados encontrados por Coledam et al. ${ }^{25}$ representam bem esta situação. Associações positivas foram encontradas entre a escolaridade do pai e o número de banheiros na residência com a $F / R$, mas associações negativas foram encontradas entre o número de televisores e o número de carros com a ApC. Esta variação nos resultados acaba não permitindo identificar de forma clara como e o quanto a condição socioeconômica influencia na ApFRS destes indivíduos.

Investigações envolvendo a zona de moradia e a ApFRS tem sido pouco realizadas. Com análises semelhantes a do presente estudo, Petroski et al. ${ }^{2}$ constataram que adolescentes da zona urbana tem chance aumentada de apresentarem baixa ApC e baixa Flex, enquanto aqueles da zona rural tem chance aumentada de apresentarem baixa F/R. No estudo de Glaner et al. ${ }^{7}$ a frequência de baixa ApFRS foi superior nos adolescentes da zona urbana nos três componentes. Embora os resultados do estudo de Glaner et al. ${ }^{7}$ estejam de acordo com a hipótese de que adolescentes da zona rural, por terem supostamente um maior gasto energético em relação àqueles da zona urbana, apresentem maior frequência de adolescentes que atendem às recomendações de ApFRS, ela não é sustentada pelos resultados de Petroski et al. ${ }^{2}$ e o presente estudo. Estes achados sugerem que os hábitos de vida de adolescentes da zona rural e sua possível associação com a ApFRS e outros desfechos em saúde devam ser mais investigados.

Quanto aos indicadores comportamentais, foram encontradas associações significativas entre não gostar de EF escolar e de esportes e a baixa ApC. A EF escolar parece ser um importante espaço para o desenvolvimento de conhecimentos práticos e teóricos que podem contribuir para um estilo de vida mais ativo e, consequentemente, para melhores níveis de ApFRS. Adolescentes que participam e gostam das aulas de EF escolar tendem a ser mais ativos ${ }^{22}$ e a apresentar melhores níveis de $\mathrm{ApC}^{11}$. Estes achados são reforçados considerando que o 
período de férias escolar parece ser negativo para os níveis de ApFRS de adolescentes, principalmente em relação à $\mathrm{ApC}^{26}$.

Para o aprimoramento dos níveis de ApFRS é necessário que os adolescentes aumentem seus NAF e optem por atividades mais ativas em detrimento daquelas que envolvem menos esforço físico. No presente estudo, adolescentes com um baixo NAF apresentaram mais chance de não atenderem às recomendações para $\mathrm{ApC}$ e F/R e aqueles com tempo de tela superior a três horas diárias apresentaram mais chance de não atenderem às recomendações para $\mathrm{ApC}$ e Flex. Outros estudos também encontraram maiores frequências de adolescentes que não atenderam às recomendações para ApFRS entre os menos ativos ${ }^{1,11,12}$ e entre aqueles com maior tempo de tela ${ }^{11,12,27}$. Não obstante, nem todos os estudos encontram estas associações ${ }^{10}$, indicando que mais investigações devem ser conduzidas analisando estas variáveis.

Em relação aos indicadores antropométricos, adolescentes com sobrepeso e com adiposidade corporal aumentada apresentaram mais chance de terem baixa ApC, e aqueles com circunferência da cintura aumentada tiveram mais chance de não atenderem às recomendações para F/R e Flex. Tais achados são coerentes com aqueles apresentados na literatura ${ }^{11-13,28,29}$, indicando que indivíduos com excesso de peso tendem a ter baixa ApFRS, agregando dois importantes fatores de risco para o desenvolvimento de doenças crônicas.

O presente estudo trouxe importantes informações para melhor compreensão dos níveis de ApFRS de adolescentes. Estas informações podem ser utilizadas para discussões e elaboração de ações para o aumento dos níveis de ApFRS desta população. Não obstante, algumas limitações devem ser apresentadas e suas repercussões discutidas.
Os resultados encontrados devem ser analisados considerando a natureza transversal dos dados que impossibilitam atribuir relação de causa e efeito às associações encontradas. Adolescentes da rede privada de ensino não fizeram parte da amostra. Considerando que o nível socioeconômico foi uma das variáveis a se associar com a baixa ApFRS, é importante que estudos envolvendo adolescentes de escolas privadas, normalmente provenientes de famílias de maior nível socioeconômico, sejam desenvolvidos. Apesar de a idade ter sido uma das variáveis estudadas, a ausência do estágio maturacional neste estudo pode ser considerada uma limitação, pois influencia nos níveis de ApFRS independente de outras variáveis inseridas neste estudo ${ }^{23}$. Por fim, a forma de medida dos componentes da ApFRS pode também ser considerada uma limitação. Mesmo os adolescentes tendo sido incentivados a desempenharem o seu melhor, durante a realização dos testes, os resultados podem ser influenciados pelo nível motivacional.

\section{CONCLUSÃO}

A prevalência de baixa ApFRS é elevada na população estudada. Os fatores associados à baixa $\mathrm{ApC}$ e à baixa $\mathrm{F} / \mathrm{R}$ muscular são similares. Porém, para a baixa Flex tendem a ser por outros fatores. Apenas os indicadores antropométricos de excesso de peso se associaram à baixa ApFRS nos três componentes.

Todos os autores declararam não haver qualquer potencial conflito de interesses referente a este artigo.

CONTRIBUIÇÕES DOS AUTORES: Cada autor contribuiu individual e significativamente para o desenvolvimento do manuscrito. TAP (0000-0002-2715-809X)* participou da concepção da proposta do estudo, das coletas de dados e da redação do manuscrito, MLBA (0000-0002-8266-0744)* participou da coleta, tabulação e análise dos dados, e da revisão da redação do manuscrito e GGB (0000-0002-6275-0232)* participou da coleta, tabulação e análise dos dados, e da redação do manuscrito. Todos os autores aprovaram a versão final do manuscrito. *Número ORCID (Open Researcher and Contributor ID).

\section{REFERÊNCIAS}

1. Guedes DP, Miranda Neto J, Lopes VP, Silva AJ. Health-related physical fitness is associated with selected sociodemographic and behavioral factors in Brazilian school children. J Phys Act Health. 2012;9(4):473-80.

2. Petroski EL, Silva AF, Rodrigues AB, Pelegrini A. Associação entre baixos níveis de aptidão física e fatores sociodemográficos em adolescentes de área urbanas e rurais. Motri. 2012;8(1):5-13.

3. Nogueira JAD, Pereira CH. Aptidão física relacionada à saúde de adolescentes participantes de programa esportivo. Rev Bras Educ Fís Esporte. 2014;28(1):31-40.

4. Petroski EL, da Silva AF, Rodrigues AB, Pelegrini A. Health-related physical fitness in Brazilian adolescents from areas having a medium/low human development. Rev Salud Publica (Bogota). 2011;13(2):219-28.

5. Guedes DP, Miranda Neto JT, Germano JM, Lopes V, Silva AJRM. Aptidão física relacionada à saúde de escolares: rograma fitnessgram. Rev Bras Med Esporte. 2012;18(2):72-6.

6. Pelegrini A, Silva DAS, Petroski EL, Glaner MF. Aptidão física relacionada à saúde de escolares brasileiros: dados do projeto esporte Brasil. Rev Bras Med Esporte. 2011;17(2):92-6.

7. Glaner MF. Aptidão física relacionada à saúde de adolescentes rurais e urbanos em relação a critérios de referência. Rev Bras Educ Fís Esporte. 2005;19(1):13-24.

8. Coledam DHC, Batista Júnior JP, Glaner MF. Baixa concordância entre os critérios de referência da fitnessgram para adolescentes. Rev Paul Pediatr. 2015;33(2):181-186.

9. Raitakari OT, Juonala M, Kähönen M, Taittonen L, Laitinen T, Mäki-Torkko N, et al. cardiovascular risk factors in childhood and carotid artery intima-media thickness in adulthood: the cardiovascular risk in young finns study. JAMA. 2003;290(17):2277-83.

10. Guedes DP, Guedes JERP, Barbosa DS, Oliveira JA. Atividade física habitual e aptidão física relacionada à saúde em adolescentes. Rev Bras Cienc Mov. 2002;10(1):13-21.

11. Coledam DHC, Ferraiol PF, dos-Santos JW, Oliveira AR. Fatores associados à aptidão cardiorrespiratória de escolares. Rev Bras Med Esporte. 2016;22(1):21-26.

12. Silva DAS, Tremblay MS, Pelegrini A, Silva JMFL, Petroski EL. Low aerobic fitness in Brazilian adolescents. Rev Bras Med Esporte. 2015;21(2):94-98.

13. Silva DAS, Nascimento TBR, Silva AF, Glaner MF. Excesso de adiposidade corporal em adolescentes: associação com fatores sociodemográficos e aptidão física. Motriz. 2013;19(1):114-25.

14. Instituto Nacional de Estudos e Pesquisas Educacionais Anísio Teixeira (Inep). Censo Escolar da Educação Básica. [Acesso em: outubro de 2010]. Disponível em http://portal.inep.gov.br/basica-censo.

15. Projeto Esporte Brasil (PROESP-Brasil). [Acesso em: outubro de 2010]. Manual. Disponível em: http:// www.proesp.ufrgs.br.

16. Associação Brasileira de Empresas de Pesquisa (ABEP). Critério de classificação econômica Brasil (dados com base no levantamento sócio econômico 2009). São Paulo: ABEP; 2011.
17. Crocker PR, Bailey DA, Faulkner RA, Kowalski KC, McGrath R. Measuring general levels of physical activity: preliminary evidence for the Physical Activity Questionnaire for Older Children. Med Sci Sports Exerc. 1997;29(10):1344-9.

18. Kowalski K, Crocker P, Faulkner R. Validation of the physical activity questionnaire for older children Pediatr Exerc Sci. 1997;9(2):174-86.

19. Cole TJ, Bellizzi MC, Flegal KM, Dietz WH. Establishing a standard definition for child overweight and obesity worldwide: international survey. BMJ. 2000;320(7244):1240-3.

20. Fernández JR, Redden DT, Pietrobelli A, Allison DB. Waist circumference percentiles in nationally representative samples of African-American, European-American, and Mexican-American children and adolescents. J Pediatr. 2004;145(4):439-44.

21. American Alliance for Health, Physical Education, Recreation and Dance (AAHPERD). Physical best. Reston, VA: AAHPERD; 1988

22. Bergmann GG, Bergmann ML, Marques AC, Hallal PC. Prevalence of physical inactivity and associated factors among adolescents from public schools in Uruguaiana, Rio Grande do Sul State, Brazil. Cad Saude Publica. 2013;29(11):2217-29.

23. Malina RM, Bouchard C, Bar-Or O. Growth, maturation, and physical activity. 2nd ed. Champaign, IL: Human Kinetics; 2004

24. Jiménez Pavón D, Ortega FP, Ruiz JR, España Romero V, García Artero E, Moliner Urdiales D, et al. Socioeconomic status influences physical fitness in European adolescents independently of body fat and physical activity: the HELENA study. Nutr Hosp. 2010;25(2):311-6.

25. Constantino-Coledam DH, Ferraiol PF, Arruda GA, Pires-Júnior R, Teixeira M, Greca JP, et al. The association between socioeconomic indicators and adolescents' physical activity and health-related fitness. Rev Salud Publica (Bogota). 2013;15(6):823-36.

26. Silva EF, Oliveira MA, Mendes EL, Ferreira AP, André R, Brito CJ, et al. Influência do período de férias na aptidão física de escolares. J Health Sci Inst. 2010;28(2):181-5.

27. Tucker JS, Martin S, Jackson AW, Morrow JR Jr, Greenleaf CA, Petrie TA. Relations between sedentary behavior and FITNESSGRAM healthy fitness zone achievement and physical activity. J Phys Act Health. 2014;11(5):1006-11.

28. Ruiz JR, Rizzo NS, Hurtig-Wennlöf A, Ortega FB, Wärnberg J, Sjöström M. Relations of total physical activity and intensity to fitness and fatness in children: the European Youth Heart Study. Am J Clin Nutr. 2006;84(2):299-303.

29. Brunet M, Chaput JP, Tremblay A. The association between low physical fitness and high body mass index or waist circumference is increasing with age in children: the 'Québec en Forme' Project. Int J Obes (Lond). 2007;31(4):637-43. 